\title{
11B-1-1
}

\section{MTRANSPARENT INSULATING CHANNELS AS COMPONENTS FOR MINIATURIZED CHEMICAL SEPARATION DEVICES}

\author{
R B.M. Schasfoort, J. Hendrikse and A. van den Berg, \\ MESA Research Institute, University of Twente \\ PO Box 217, 7500 AE Enschede. The Netherlands \\ Tel.+31534892691,Fax.+31534892287,E-mail: A.vandenBerg@el.utwente.nl
}

\begin{abstract}
Currently, miniaturized devices that apply electro osmotic pumping or electrophoretic separations are mostly constructed by etching small insulating channels for supply and separation on glass substrates. In principle, silicon is a superior construction material in terms of inertness and design flexibility. However, because of its semiconducting properties, the use in high voltage applications like the ones mentioned above is quite limited.

In this paper, the use of $\mu$ Transparent Insulating Channel $(\mu$ TIC) technology is demonstrated as a standard procedure to manufacture miniaturized analytical separation devices. This technique, $\mu$ channels having extremely thin, transparent and insulating walls can be fabricated. An overview of the impact of this technology is given, showing the advantages of a fabrication technology that is as flexible as silicon technology for the fabrication of $\mu$ TAS or "lab on a chip" devices. The following basic technology and control parameters will be highlighted.
\end{abstract}

1. Up to $100 \mu \mathrm{m}$ wide rectangular channels

2. Bosses and leak-free connections to external $\mu$ fluidics.

3. Web-like structures for inlets/outlets $>100 \mu \mathrm{m}$.

4. Implementation of conductivity electrodes

5. Good thermal dissipation properties of the thin walls

6. Control of the electro osmotic flow by a radial voltage

\section{INTRODUCTION}

Recently a new fabrication method to produce miniaturized Transparent Insulating Channels ( $\mu$ TICs) has been presented by Tjerkstra [1]. $\mu$ TICs allow high flexibility in the design of insulating channels with respect to dimensions and crosssectional shapes for analytical techniques and a re made by a standard two mask process in the MESA institute. The process leaves insulating, very thin walled and optically transparent channels. It is schematically shown in figure 1 .

\subsection{Fabrication Process}

CHANNELS Defines the channel geometry in a silicon wafer that is being etched by Deep Reactive Ion Etching (DRIE).

KOH ETCH Defines the boss geometry and the sacrificial $\mathrm{Si}$ etch area.

The two masks needed for the process are shown in the figure:

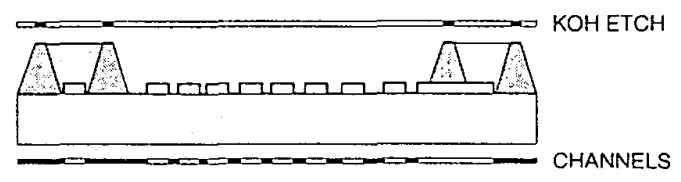

Figure 1: Schematic presentation of the process and masks

The shape of the $\mu$ TICs is defined by Deep Reactive Ion Etching (DRIE) of a silicon substrate (dark grey). A thin insulating silicon nitride film that is to be the channel wall of the $\mu$ TIC is deposited on the silicon. The structure is fixed by anodic bonding to a second, glass, wafer (light grey). Subsequently, the silicon is back etched, except in the parts where the bosses described below are needed. This process is the basis for the building blocks and unit operations described in the remainder of this paper.

\section{REALIZED $\mu$ TICS: DESIGN FLEXIBILITY}

\subsection{Channels}

Capillary Ion Electrophoresis (CIE) is widely applied in analytical chemistry [2]. In order to apply high voltages for the separation process, highly insulating, narrow channels are needed. Any length or geometry including curves and junctions can be manufactured using our standard process. The channels presented in this paper have a height of $25 \mu \mathrm{m}$. The width (default $25 \mu \mathrm{m}$ ) is 
dependent on the specifications for the application[3].

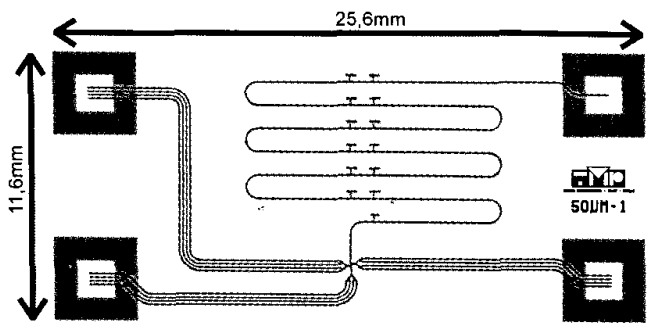

Figure 2: Layout of the current $\mu$ TIC structure (above) and SEM-pictures showing the double Tinjector in various degrees of detail various details (below).

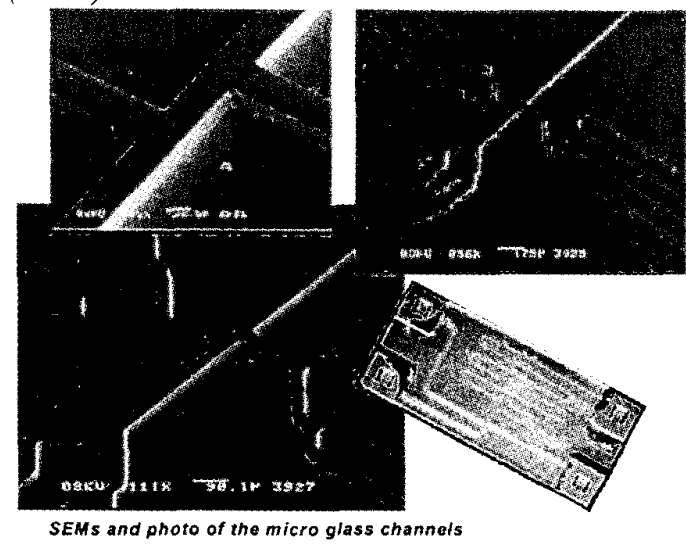

\subsection{Bosses}

In order to connect a (mini)fluidic handling system to the device, $6 \times 6 \mathrm{~mm}^{2}$ silicon fluid inlets and outlets were processed on the chip, as indicated in Fig. 1. These so called bosses occupy a relatively large area of the chip and should therefore be effectively positioned. A cuvette with $3-4 \mathrm{~mm}$ diameter $O$ rings has been used to leak-free connect the bosses to an external fluid handling cuvette system that enables fast computer controlled filling of the devices. Moreover, problems concerning air entrapment in the channels or drying by evaporation are circumvented with this fluid handling system.

\subsection{Web-like structures}

Using current technology, the maximum obtainable width of $\mu$ TICs is approximately $100 \quad \mu \mathrm{m}$.

Wider channels will break because of mechanical stress in the material and fragility of the large overhang. To overcome this problem, the web-like structure shown in Fig 3 has been fabricated.

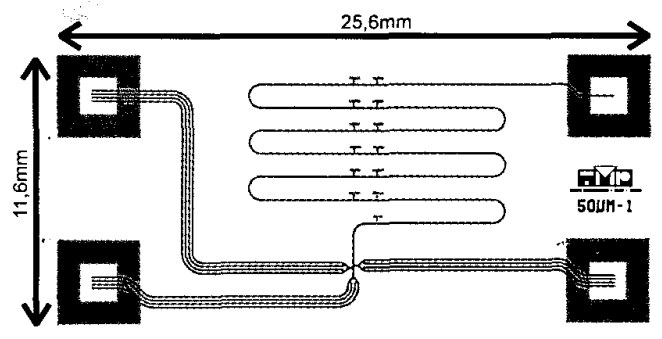

Figure 3: Optical Micrograph of a Web-like microchannel structure.

These grids of channels with pillars in between can cover a large area $(\gg 100 \mu \mathrm{m}$ in width). Filling of the structure by capillary flow was no problem when the solution had to move from a high number of channels into towards the single channel. However, when the structure was filled from the other side, entrapment of stable air bubbles occurred. Electro osmotic flow in the web appears to be even along its entire cross section: when Rhodamine B was introduced as a dye during the generation of EOF, a single straight front was observed.

Using this method, any channel width can be processed without breaking the thin channel walls. This structure can be used to lower the impedance of inlet channels as well as function as a highly reproducible stationary phase micro chromatography column, comparable to the collocated monolith support structures proposed by He et al. [4]

\subsection{Metal $\mu$ contacts as electrodes}

While on the one hand the transparency of the channel walls is compatible with optical detection schemes, the use of electrochemical detection methods is being investigated as well at our institute. On-chip electrochemical detection offers excellent detection limits and a great wealth of detection schemes. $[5,6]$ Platinum electrodes in the $\mu$ TICs were made by metal deposition and photolithography on the glass substrate. Titanium was used as a connecting layer and in order to minimize problems caused by the thickness of these electrodes during the anodic bonding stage, slight cavities were etched in the glass wafer prior to the electrode deposition step. In this way, onchip two- and four point conductivity detection 
was implemented. Exact positioning during the anodic bonding step is important to position the electrodes in the channels with good liquid contact. An $8 \mu \mathrm{m}$ width to $8 \mu \mathrm{m}$ gap conductivity electrode centered in a $24 \mu \mathrm{m}$ channel was realized. The electrolyte resistance of a $1 \mathrm{mM} \mathrm{KCl}$ solution was measured at $1 \mathrm{kHz}$ using Lock-in amplifier techniques and appeared to be ca $2 \mathrm{M} \Omega$.

\section{REALIZED $\mu$ TICS: EXPLOITING THE SMALL WALL THICKNESS}

The small wall thickness that can be realized by the $\mu \mathrm{TIC}$ technology has a number of advantages of its own, apart from the design flexibility mentioned above. Fig. 4 shows a scanning electron micrograph of a $\mu$ TIC that is cleaved perpendicular to the channel axis. The minimum possible wall thickness is determined by the structural integrity of the channel while its maximum is determined by the need for an anodic bonding step at the end of the fabrication process. An optimum thickness of $350 \mathrm{~nm}$ is used for the fabrication of the structures described in this paper.

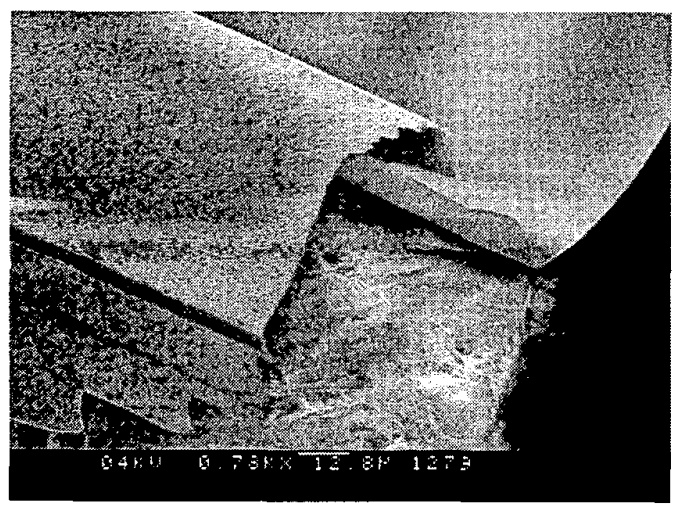

Figure 4: SEM-picture of a $\mu T I C$, perpendicularly cleaved to the channel axis.

\subsection{Joule Heating}

In electrophoretic separations, high field strengths allow far fast separation and minimize band broadening by diffusion. However, the high currents connected to these high fields lead to heating, which increases diffusion effects. While capillaries can be cooled using a number of methods, channels that are entrapped in glass chips are less easy to cool. In contrast, the very thin channel walls of the $\mu$ TICs, the device can be cooled very easily and therefore can be effectively temperature controlled. This more effective heat dissipation enables the use of higher electric field strengths leading to faster and better separations. Moreover, samples having a higher ionic strength can be separated. For the preliminary measurements presented here, the channels were filled with a $1: 150 \mathrm{mM} \mathrm{KCL/ethanol} \mathrm{solution.}$ Relatively low voltages (up to $1200 \mathrm{~V}$ were applied for safety reasons and the current through the channels was measured. The results are shown in Fig 5. Usually, excessive Joule heating is said to occur when a the I-V curve diverts from the straight line predicted by Ohm's law. This indicates a higher conductivity of the fluid in the channel and thus a higher temperature. The same measurements were repeated while a small fan was directed at the $\mu$ TIC. In Fig. 5, it can clearly be seen that the current in the actively cooled channel remains closer to its ohmic value than the current through the channel to which no cooling is applied. Currently, efforts are under way to determine the effective temperature of the buffer solution in the device from the relation between buffer conductivity and temperature in order to calculate the heat dissipation per unit of length in the channel.

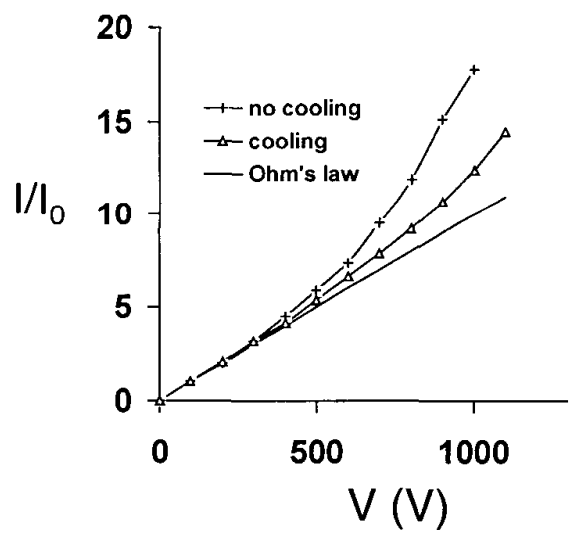

Figure 5: Measured current divided by the current at $V=100 \mathrm{~V}$ as a function of applied voltage, $V$. Note that all curves appear to be more or less ohmic at voltages below $500 \mathrm{~V}$

\subsection{Electro Osmotic Flow control using a radial electric field}

The $\mu$ TIC process was also shown to be effective to control the EOF with an external radial electric 
field $[7,8]$. As illustrated in Fig 6, the external field modifies the zeta potential of the channel wall, which in turn changes the magnitude, or even direction, of the EOF.
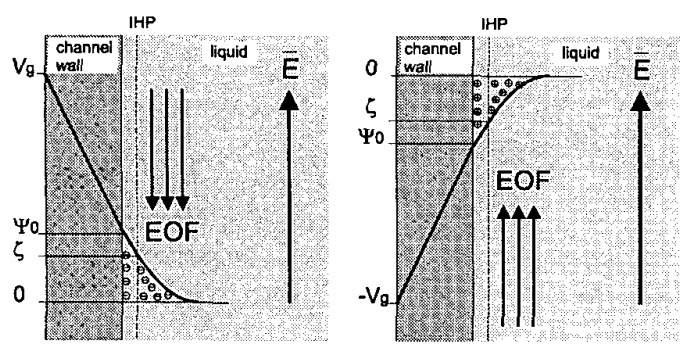

Figure 6: Illustration of the influence of an external field to the channel wall on the zeta potential $\zeta$ and EOF. Note that $V_{g}$ is proportional to the channel wall thickness.

While this phenomenon has been demonstrated in capillaries, the conventional glass chip format of electrophoresis chips is not fit to implement this feature. The structure consists of an inlet and an outlet boss connected by a single $\mu$ TIC channel At the middle of the channel a free-standing silicon "gate" was positioned with only capacitive coupling to the electrolyte solution (see figure 5). Transport of the electrolyte is carried out applying EOF (electro osmotic flow) in the channels. Our observations showed that the radial electric field can change the zeta potential of the channel wall in that way that even the direction of the flow was reversed. From Fig. 6 it is clear that the gate voltage that needs to be applied to achieve the effect is proportional to the wall thickness. Due to the small thickness of the channel wall, only several tens of volts were needed. When voltages over $150 \mathrm{~V}$ were applied to the gate, breakdown occurred, in accordance with findings on early Sibased electrophoresis devices.[9]

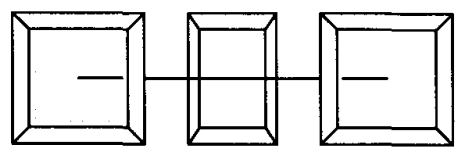

Boss A Silicon Boss B

Figure 7: Schematic drawing of the device that was used to apply an external radial field on the channel. EOF from Boss $A$ to Boss $B$ can be controlled by the potential applied to the silicon.

\section{CONCLUSION}

An overview is given of the potential of structures based on the $\mu$ TIC technology. An effective connection technology employing silicon bosses is briefly described and the possibility to fabricate complicated geometries is demonstrated by the web-structures shown in Fig 3. On-chip electrochemical sensors have successfully been integrated. The thin walls are demonstrated to be advantageous for effective cooling of the devices as well as the modulation of zeta potentials. By designing structures for miniaturized Total Analysis Systems ( $\mu$ TAS). or "lab on the chip" applications these structures and control parameters are now standard tools in our institute.

\section{REFERENCES}

[1] Tjerkstra, R.W., De Boer, M., Berenschot, E., Gardeniers, J.G.E., Van den Berg, A.,Elwenspoek, M.C., Etching technology for microchannels, Proc. MEMS 97 (1997), 147-152.

[2] J.P. Landers, "Handbook of Capillary Electrophoresis", $2^{\text {nd }}$ Edition, CRC Press, New York, (1997).

[3] Y. Fintschenko, Patrick Fowler, Vincent Spiering, Gert-Jan Burger, and Albert van den Berg , "Characterization of Silicon-based Insulating Channels for Capillary Electrophoresis", Proc. $\mu$ TAS '98, (1998), 327-331.

[4] B. He, N. Tait, F. Regnier, Fabrication of Nanocolumns for Liquid Chromatography, Anal. Chem. 70(18),3790-3797, (1998) .

[5] R.J. Reay at al G.T.A. Kovacs Microfabricated EC detector for CE, Proceedings of the Solid State S\&A workshop South Carolina, June 1994.

[6] A.T. Woolley, K. Lao, A.N. Glazer, R.A. Mathies, "Capillary Electrophoresis Chips with Integrated Electrochemical Detection" Anal. Chem. 70(4), 684-688 (1998).

[7] M.A. Hayes and A.G.Ewing Electroosmotic Flow Control and Monitoring with an applied Radial voltage for Capillary Zone Electrophoresis. Anal. Chem. 1992, 64, 512-516.

[8] V. Kašièka, Z Prusík, P. Sázelová, T. Barth, E. Brynda, L Machová. Capillary zone electrophoresis with external radial electric field control of electroosmotic flow and its application to the separation of synthetic oligopeptides. Journal of Chromatography A 772 (1997) 221-230 [9] D.J. Harrison, P.G. Glavina, Towards Miniaturized Electrophoresis and Chemical 
Analysis Systems on Silicon: an Alternative to

Chemical Sensors, Sens. Actuators B 10, 107-116 (1993).

\section{ACKNOWLEDGEMENT}

The authors wish to thank Stephan Schlauchman for fabrication of the devices presented in this paper 